\title{
Associação entre Perfil Lipídico e Adiposidade Corporal em Mulheres com Mais de 60 Anos de Idade
}

\author{
Association Between Lipid Profile and Adiposity in Women Over Age 60 \\ Maressa Priscila Krause, Tatiane Hallage, Mirnaluci P. R. Gama, Jeffer Eidi Sasaki, Cristiane Petra Miculis, Cosme Franklin \\ Buzzachera, Sergio Gregorio da Silva \\ Universidade Federal do Paraná - Faculdade Evangélica do Paraná - Curitiba, PR - Brasil
}

\section{Resumo}

Objetivo: Verificar a associação entre perfil lipídico e medidas de obesidade corporal global e central em mulheres com idade superior a 60 anos.

Métodos: A amostra foi composta por 388 mulheres, com mais de 60 anos de idade (média, 69,0; desvio padrão, 5,9 anos). O perfil lipídico foi determinado por meio das dosagens de colesterol total (CT), colesterol de lipoproteína de alta densidade (HDL-colesterol), colesterol de lipoproteína de baixa densidade (LDL-colesterol) e triglicerídeos (TG). A obesidade global foi mensurada pelo índice de massa corporal (IMC) e pelas dobras cutâneas (DC), e a obesidade central foi mensurada pela circunferência da cintura (CC) e pela relação cintura-quadril (RCQ). A análise estatística foi realizada por meio da correlação parcial ajustada para a idade e ANOVA one-way $(p<0,05)$.

Resultados: Os valores médios encontrados nas variáveis de adiposidade corporal e nos componentes do perfil lipídico indicam elevado risco aterogênico. Além disso, os indicadores de obesidade tanto global como central foram diretamente associados com os níveis de TG e inversamente associados com os níveis de HDL-colesterol.

Conclusão: A análise de correlação parcial e a maior variância encontrada na CC e na RCQ com os componentes do lipidograma sugerem que ambos os métodos podem auxiliar no diagnóstico precoce da aterosclerose. (Arq Bras Cardiol 2007;89(3):163-169)

Palavras-chave: Adiposidade, fatores de risco, aterosclerose, mulheres.

Summary
Objective: To verify the association between lipid profiles and overall or central obesity in women over the age of 60.

Methods: The sample was comprised of 388 women over the age of 60 (mean 69; standard deviation 5.9 years). The lipid profile was determined using total cholesterol (TC), HDL cholesterol (HDL-C), LDL cholesterol ( $L D L-C)$ and triglyceride (TG) levels. Overall obesity was determined using the body mass index (BMI) and skin fold (SF) measurements. Central obesity was determined using the waist circumference (WC) and waist - hip ratio (WHR). Statistical analysis was conducted using age adjusted partial correlation and one way ANOVA $(p<0.05)$.

Results: The mean values found for the adiposity variables and lipid profile components indicate an elevated atherogenic risk. In addition, the indicators for overall and central obesity were directly related to TG levels and inversely related to HDL-C levels.

Conclusion: The partial correlation analysis and the largest variance found for WC and WHR in comparison to the lipidogram components indicate that both methods could be useful in the early diagnosis of atherosclerosis. (Arq Bras Cardiol 2007;89(3):147-153)

Key words: Adiposity; risk factors; atherosclerosis; women.

Full texts in English - http://www.arquivosonline.com.br

\section{Introdução}

As doenças cardiovasculares, principalmente as doenças coronarianas, são as principais causas de mortalidade em todo o mundo ${ }^{1,2}$. A aterosclerose ou doença aterosclerótica atinge vasos sanguíneos periféricos e centrais, podendo ser considerada um

Correspondência: Maressa Priscila Krause •

Rua José Rodrigues Pinheiro, 949 - 81130-200 - Curitiba, PR - Brasil

E-mail: maressakrause@hotmail.com

Artigo recebido em 26/07/06; revisado recebido em 26/03/07; aceito em

24/04/07. estado inflamatório ativo, e é caracterizada por alterações em marcadores inflamatórios e em fatores de risco ${ }^{3,4}$.

Vários fatores de risco para o desenvolvimento aterogênico têm sido descritos, entre eles aumento dos níveis de triglicerídeos (TG), de colesterol total (CT) e de colesterol de lipoproteína de baixa densidade (LDL-colesterol), diminuição do colesterol de lipoproteína de alta densidade (HDLcolesterol), hipertensão, diabetes, tabagismo e inatividade física ${ }^{4,5}$. Dentre esses fatores, o perfil lipídico tem sido amplamente estudado pela sua elevada associação com a 
aterogênese ${ }^{6-9}$. Similarmente, a obesidade corporal também tem sido investigada como fator de risco para as doenças ateroscleróticas e cardiovasculares ${ }^{10-15}$.

A obesidade central tem demonstrado predizer todas as causas de mortalidade em mulheres idosas brasileiras, como também elevar o risco para futuros eventos cardiovasculares ${ }^{14,15}$. Esses resultados indicam a importância da investigação de como esses indicadores antropométricos podem ser utilizados como instrumento de identificação de indivíduos com elevada propensão para a aterosclerose.

A adiposidade corporal pode ser avaliada a partir de métodos antropométricos que possuem diversos benefícios, por seu caráter não-invasivo, baixo custo operacional, fácil aplicabilidade, e ampla utilização em meios clínicos e estudos epidemiológicos, sendo caracterizados como uma forma de avaliação indireta para riscos à saúde ${ }^{16-18}$.

Os métodos antropométricos mais utilizados para a avaliação do acúmulo de massa gorda são a circunferência da cintura (CC), a relação cintura-quadril (RCQ), o índice de massa corporal (IMC) e as dobras cutâneas (DC). Apesar de diversos estudos verificarem a associação entre risco aterogênico com medidas antropométricas e perfil lipídico, os resultados que explicariam de que forma as variáveis antropométricas se associam com o perfil lipídico não são completamente conclusivos ${ }^{10,11,19}$.

A associação entre fatores de risco para doença aterosclerótica diretos (perfil lipídico) e indiretos (variáveis antropométricas) para mensurar o acúmulo de massa gorda não é, portanto, totalmente clara para auxiliar o diagnóstico clínico desse quadro. Sendo assim, o objetivo deste estudo foi verificar a associação entre perfil lipídico (CT, TG, LDLcolesterol, HDL-colesterol) e medidas de obesidade global (IMC e DC) e de obesidade central (CC e RCQ) em mulheres com mais de 60 anos de idade.

\section{Métodos}

O delineamento da pesquisa é caracterizado como Estudo Observacional Transversal, Descritivo e Correlacional. A coleta de dados foi realizada no período compreendido entre abril e julho de 2006.

População e amostra - Com o intuito de realizar uma seleção de amostra estratificada, as seguintes etapas foram realizadas: 1) Cadastro dos grupos comunitários existentes no município de Curitiba, Paraná, obtido mediante parceria com instituições que promovem atividades recreacionais para a população da respectiva regional geográfica. 2) Mapeamento de todos os grupos cadastrados nas oito regionais do município. 3) Alocação aleatória simples dos grupos que seriam convidados a participar da pesquisa, por regional. 4) Visita ao grupo, explicação dos procedimentos da pesquisa, e convite à participação voluntária no estudo para os indivíduos pertencentes ao respectivo grupo comunitário, extensivo a seus familiares e amigos próximos.

Depois de realizado o procedimento de seleção amostral, conforme descrito, foi determinado o cronograma para a coleta de dados. A amostra foi constituída de 388 mulheres (média de idade, 69,0; desvio padrão, 5,9 anos) que estivessem, na data da coleta, com idade cronológica superior ou igual a
60 anos. Entretanto, foi obtido n = 383 para a variável LDLcolesterol, em decorrência da exclusão de 5 sujeitos que apresentaram valores de TG superiores a 400,0 mg/dl.

A amostra foi constituída predominantemente de indivíduos da cor branca e de nível socioeconômico baixo (76,3\%). Desses indivíduos, apenas 23,4\% relataram realizar tratamento medicamentoso para dislipidemia e 13,3\%, para diabetes. Quase metade da amostra $(45,7 \%)$ apresentou antecedente de doença coronariana em pai, mãe ou irmão(ã), 4,4\% eram tabagistas e $63,3 \%$ praticavam algum tipo de exercício físico regular (no mínimo duas horas semanais).

Após detalhado esclarecimento sobre os propósitos dessa investigação, os procedimentos utilizados, e os benefícios e os possíveis riscos atrelados, os sujeitos participantes assinaram o termo de consentimento, condicionando sua participação de modo voluntário. O protocolo de pesquisa foi aprovado pelo Comitê de Ética do Setor de Ciências Biológicas da Universidade Federal do Paraná, conforme as normas estabelecidas na Declaração de Helsinque e na Resolução 196/96 do Conselho Nacional de Saúde sobre pesquisa envolvendo seres humanos.

Instrumentos e procedimentos - Com o objetivo de evitar a influência de variações circadianas, todas as avaliações foram realizadas no mesmo período do dia (entre $8 \mathrm{~h} 00$ e 10h00). Além disso, os sujeitos participantes foram instruídos a não realizar atividade física vigorosa no dia anterior, como também a não ingerir alimento nas duas horas que antecederam o início da avaliação. As avaliações foram realizadas no Departamento de Educação Física Laboratório de Fisiologia do Centro de Pesquisa em Exercício e Esporte, da Universidade Federal do Paraná.

As variáveis antropométricas foram obtidas conforme os procedimentos propostos por Lohman e cols. ${ }^{20}$.

Para a determinação da estatura corporal (Est, cm), o indivíduo avaliado deveria permanecer em posição ortostática, com os pés unidos, descalços, utilizando o mínimo possível de roupas. Além disso, deveria manter-se em apnéia inspiratória e com a cabeça orientada em 90 graus, conforme plano de Frankfort, tendo as superfícies de calcanhar, cintura pélvica, cintura escapular e região occipital em contato com o estadiômetro (SANNY, modelo STANDARD, precisão de $0,1 \mathrm{~cm})$, fixado à parede.

A massa corporal $(\mathrm{MC}, \mathrm{kg}$ ) foi mensurada com o indivíduo avaliado permanecendo em posição ortostática, descalço, e trajando o mínimo possível de roupas. A MC deveria permanecer igualmente distribuída entre os membros inferiores durante a permanência na plataforma da balança (TOLEDO, modelo 2096 PP, precisão de 0,1 kg).

O IMC foi obtido mediante a utilização do quociente massa corporal/estatura2, em que o valor da MC é expresso em kg e o da Est, em $\mathrm{cm}^{2,21}$.

A CC $(\mathrm{em} \mathrm{cm})$ foi mensurada no ponto médio entre a crista ilíaca e a face externa da última costela. A circunferência do quadril foi mensurada na maior circunferência da região glútea, com o avaliador posicionado ao lado do avaliado. Como instrumento de medida foi utilizada fita antropométrica inelástica (precisão de 0,1 cm).

A RCQ foi calculada dividindo-se o valor da CC $(\mathrm{em} \mathrm{cm})$ pelo valor da circunferência do quadril $(\mathrm{em} \mathrm{cm})^{21}$. 
As DC foram mensuradas com o compasso de DC do tipo Lange (aproximação de 0,5 mm) nos seguintes pontos anatômicos: tríceps, abdome, supra-ilíaca, coxa medial e panturrilha medial, todos avaliados no hemicorpo direito ${ }^{22}$.

Preconizando evitar as variações inter-avaliadores, a avaliação de cada variável antropométrica (MC, Est, CC e DC) foi realizada pelo mesmo avaliador, previamente treinado, em todos os sujeitos.

Para avaliação dos níveis de TG e CT, foi utilizado o método enzimático. O HDL-colesterol foi mensurado por meio do sistema de precipitação seletiva da LDL e da lipoproteína de muito baixa densidade (VLDL). Após a centrifugação, o HDL-colesterol foi determinado no sobrenadante, com a utilização do método enzimático. Todas as medidas foram analisadas no espectrofotômetro COBAS MIRA PLUS (Roche Diagnostics), com calibrador e soro controle DIASYS. O kit da LABTEST foi utilizado para a realização das dosagens de CT e TG, enquanto para a dosagem do HDL-colesterol utilizou-se o kit DIASYS. O LDL-colesterol foi calculado de acordo com a fórmula de Friedewald ${ }^{23}$, em que LDL-colesterol $=\mathrm{CT}$ - (HDL-colesterol + TG/5). Foram excluídos 5 indivíduos da amostra total para a determinação do LDL-colesterol, pelo fato de o valor de TG ter se apresentado superior a 400,0 md/dl.

Análise estatística - A normalidade da distribuição das variáveis foi determinada a partir do teste de KolmogorovSmirnov. Subseqüentemente, foram aplicadas medidas de tendência central e variabilidade para a análise descritiva dos dados. A correlação parcial ajustada para a idade foi utilizada a fim de verificar o grau de associação entre as variáveis antropométricas e os componentes do perfil lipídico, enquanto a análise de variância (ANOVA) foi utilizada para verificar diferenças entre as medidas de obesidade central (CC e RCQ) de acordo com os pontos de corte do perfil lipídico.

A análise dos dados do presente estudo foi realizada mediante a utilização do Statistical Package for the Social Sciences (SPSS, versão 13.0) for Windows.

\section{Resultados}

A Tabela 1 apresenta os valores descritivos das variáveis estudadas. A partir da média do IMC e da CC, os sujeitos foram classificados como sobrepeso. A RCQ também demonstrou valores superiores à normalidade. Na análise da média dos valores encontrados no lipidograma, o componente que demonstra alterações elevadas em relação à normalidade é o HDL-colesterol.

As análises das associações entre as variáveis antropométricas estudadas, relacionadas à obesidade corporal, e o perfil lipídico estão apresentadas na Tabela 2. A obesidade corporal foi associada diretamente com os níveis de TG, enquanto essa associação com o HDL-colesterol foi inversa, independentemente do procedimento de medida antropométrica utilizado.

As Tabelas 3, 4, 5 e 6 apresentam a média da CC e da RCQ de acordo com os pontos de referência relacionados ao aumento de risco à saúde, em cada componente do lipidograma.

A CC demonstrou maior variabilidade nos pontos de corte para os TG, enquanto a RCQ apresentou diferenças entre os pontos de referência extremos. Contudo, tanto a CC como a RCQ demonstraram variância similar em relação ao HDLcolesterol. A média da CC e da RCQ no CT e no LDL-colesterol não revelou alterações significantes.

\section{Discussão}

A obesidade tem sido considerada uma epidemia mundial ${ }^{24,25}$. O excesso de gordura corporal eleva os riscos para doenças por todas as causas, incluindo as doenças cardiovasculares $12,14,15,17,26,27$. As mulheres deste estudo apresentaram condições de sobrepeso global e central, de acordo com os pontos de corte estabelecidos para IMC $\geq$ 25,0 e $<30,0$ e CC $\geq 80 \mathrm{~cm}$ e $\leq 88 \mathrm{~cm}^{28,29}$. Sendo assim,

\begin{tabular}{|c|c|c|c|c|}
\hline \multicolumn{5}{|c|}{ Tabela 1 - Valores descritivos das variáveis estudadas } \\
\hline Variável & Média & Desvio padrão & Mínimo & Máximo \\
\hline Idade (anos) & 69,0 & 5,9 & 60,0 & 87,5 \\
\hline MC (kg) & 67,7 & 11,1 & 39,0 & 110,0 \\
\hline Est (cm) & 154,6 & 6,6 & 132,0 & 180,0 \\
\hline IMC (kg/m²) & 28,3 & 4,4 & 17,4 & 44,6 \\
\hline Cintura (cm) & 86,4 & 9,5 & 63,0 & 114,0 \\
\hline RCQ & 0,85 & 0,07 & 0,30 & 1,10 \\
\hline$\sum \mathrm{DC}(\mathrm{mm})$ & 154,7 & 34,0 & 52,0 & 254,0 \\
\hline TG (mg/dl) & 139,3 & 66,9 & 32,8 & 395,2 \\
\hline CT (mg/dl) & 197,3 & 50,1 & 72,7 & 390,3 \\
\hline HDL-colesterol (mg/dl) & 47,3 & 12,5 & 20,1 & 87,0 \\
\hline LDL-colesterol (mg/dl) & 122,2 & 44,0 & $-48,0$ & 309,2 \\
\hline
\end{tabular}


Tabela 2 - Associação entre obesidade corporal e componentes do lipidograma

\begin{tabular}{lcc} 
Correlação parcial - controlada pela idade & & $\mathbf{p}$ \\
\hline IMC X TG & 0,147 & 0,004 \\
\hline IMC X CT & 0,011 & 0,831 \\
\hline IMC X HDL-colesterol & $-0,155$ & 0,002 \\
\hline IMC X LDL-colesterol & 0,012 & 0,819 \\
\hline CC X TG & 0,200 & $<0,001$ \\
\hline CC X CT & 0,021 & 0,680 \\
\hline CC X HDL-colesterol & $-0,237$ & $<0,001$ \\
\hline CC X LDL-colesterol & 0,031 & 0,547 \\
\hline RCQ X TG & 0,198 & $<0,001$ \\
\hline RCQ X CT & $-0,026$ & 0,619 \\
\hline RCQ X HDL-colesterol & $-0,242$ & $<0,001$ \\
\hline RCQ X LDL-colesterol & $-0,020$ & 0,692 \\
\hline LDC X TG & 0,108 & 0,036 \\
\hline EDC X CT & 0,017 & 0,738 \\
\hline EDC X HDL-colesterol & $-0,120$ & 0,019 \\
\hline EC X LDL-colesterol & 0,021 & 0,680 \\
\hline \hline
\end{tabular}

IMC - índice de massa corporal; TG - triglicerídeos; CT - colesterol total; HDL-colesterol - colesterol de lipoproteína de alta densidade; LDL-colesterol - colesterol de lipoproteína de baixa densidade; CC - circunferênia da cintura; RCQ - relação cintura-quadril; $\Sigma D C$ - somatório das dobras cutâneas.

Tabela 3 - Descrição da variável triglicerídeos - média e desvio padrão (entre parênteses), de acordo com as referências de risco à saúde

\begin{tabular}{lccc} 
Triglicerídeos $(\mathbf{m g} / \mathbf{d l})$ & $\mathbf{1 5 0 , 0}(\mathbf{n}=\mathbf{2 4 8})$ & $>\mathbf{1 5 0 , 0} \mathbf{e} \mathbf{2 0 0 , 0}(\mathbf{n}=\mathbf{6 8})$ & $>\mathbf{2 0 0 , 0}(\mathbf{n}=\mathbf{7 2})$ \\
\hline Idade (anos) & $68,8(5,9)$ & $69,5(5,6)$ & $68,7(5,9)$ \\
CC $(\mathrm{cm})$ & $85,0(9,5)$ & $88,3(9,9)^{*}$ & $89,5(8,3)^{*}$ \\
RCQ & $0,84(0,07)$ & $0,86(0,08)$ & $0,87(0,05)^{*}$ \\
\hline
\end{tabular}

* Diferente da categoria < 150,0; $p<0,05 ; n$ - número de pacientes; CC - circunferência da cintura; RCQ - relação cintura-quadril.

Tabela 4 - Descrição da variável colesterol total - média e desvio padrão (em parênteses), de acordo com as referências de risco à saúde

\begin{tabular}{lcccc}
$\begin{array}{l}\text { Colesterol total } \\
(\mathbf{m g} / \mathbf{d l})\end{array}$ & $\mathbf{1 8 0 , 0}(\mathbf{n}=\mathbf{1 6 0})$ & $\boldsymbol{> 1 8 0 , 0} \mathbf{e}<\mathbf{2 0 0 , 0}(\mathbf{n}=\mathbf{6 0})$ & $\mathbf{> 2 0 0 , 0} \mathbf{e}<\mathbf{2 4 0 , 0}(\mathbf{n}=\mathbf{9 3})$ & $>\mathbf{2 4 0 , 0}(\mathbf{n}=\mathbf{7 5})$ \\
\hline Idade (anos) & $69,1(6,1)$ & $68,5(6,0)$ & $68,9(5,4)$ & $68,9(5,9)$ \\
CC $(\mathrm{cm})$ & $86,4(8,8)$ & $87,0(10,8)$ & $86,0(9,5)$ & $86,5(10,0)$ \\
RCQ & $0,86(0,06)$ & $0,86(0,06)$ & $0,83(0,09)$ & $0,85(0,07)$ \\
\hline
\end{tabular}

n - número de pacientes; CC - circunferência da cintura; RCQ - relação cintura-quadril.

parece que a maioria dessas mulheres poderia apresentar risco indireto aumentado para aterosclerose.

Essa relação entre obesidade e risco cardíaco deve ser analisada cautelosamente, pois, segundo Romero-Corral e cols. ${ }^{30}$, em recente estudo meta-analítico, a obesidade global, determinada pelos valores de IMC entre $25,0 \mathrm{~kg} / \mathrm{m}^{2}$ e 30,0 $\mathrm{kg} / \mathrm{m}^{2}$, indicou a diminuição do risco para a ocorrência de eventos cardiovasculares, sugerindo um efeito cardioprotetor para a condição geral de sobrepeso. Contudo, a investigação realizada por Cabrera e cols. ${ }^{15}$ demonstrou que a obesidade 


\begin{tabular}{|c|c|c|c|}
\hline HDL-colesterol (mg/dl) & $<40,0(n=107)$ & $>40,0$ e $<65,0(n=243)$ & $>65,0(n=38)$ \\
\hline Idade (anos) & $69,1(6,0)$ & $68,7(5,8)$ & $70,2(6,0)$ \\
\hline $\mathrm{CC}(\mathrm{cm})$ & $88,4(7,9)$ & $86,1(9,7)$ & $82,6(11,1)^{*}$ \\
\hline RCQ & $0,86(0,06)$ & $0,85(0,08)$ & $0,82(0,07)^{*}+$ \\
\hline
\end{tabular}

\begin{tabular}{|c|c|c|c|}
\hline LDL-colesterol (mg/dl) & $<100,0(n=124)$ & $>100,0$ e $<130,0(n=119)$ & $>130,0(n=140)$ \\
\hline Idade (anos) & $69,7(6,3)$ & $68,3(5,6)$ & $68,9(5,7)$ \\
\hline $\mathrm{CC}(\mathrm{cm})$ & $85,6(8,9)$ & $86,9(10,1)$ & $86,6(9,6)$ \\
\hline RCQ & $0,85(0,06)$ & $0,86(0,08)$ & $0,84(0,07)$ \\
\hline
\end{tabular}

central, representada pela $R C Q$, prediz futuros eventos cardiovasculares em mulheres de 60 a 84 anos de idade, indicando risco relativo de 1,72 para valores de RCQ superiores a 0,98 (hazard ratio $=1,72$; intervalo de confiança de $95 \%=1,05-2,82 ; p=0,03)$.

Os riscos aterogênicos também podem ser determinados pelas alterações em fatores de risco direto, como o lipidograma ${ }^{31,32}$. Segundo a III Diretrizes Brasileiras sobre Dislipidemias $^{33}$ e Souza e cols. ${ }^{31}$, os seguintes pontos de corte elevam o risco para esse quadro clínico: TG acima de 150,0; CT acima 180,0; HDL-colesterol abaixo de 65; e LDL-colesterol acima de 100,0. Dessa forma, os valores médios encontrados nos componentes do perfil lipídico (Tab. 1) também demonstram que as mulheres apresentam esse fator de risco elevado.

A associação entre fatores de risco indireto e direto pode auxiliar o diagnóstico clínico. Hu e cols. ${ }^{34}$ examinaram a associação entre as alterações no lipidograma e a obesidade corporal, em mulheres com 45 a 74 anos de idade, demonstrando que tanto a obesidade global, representada pelo IMC, como a obesidade central, representada pela CC, apresentaram-se inversamente associadas ao HDL-colesterol $(r=-0,024$ e $r=-0,23[p<0,001]$, respectivamente) e ao LDL-colesterol $(r=-0,08[p<0,05]$ e $r=-0,11[p<$ $0,001]$, respectivamente). A CC, por sua vez, só foi associada diretamente com os níveis de TG $(r=0,14 ; p<0,001)$.

Outro método de avaliação da obesidade global avaliada no presente estudo foi o somatório de dobras cutâneas ( $\left.\sum \mathrm{DC}\right)$. Contudo, parece que as alterações da distribuição da gordura corporal relacionadas ao avanço da idade podem não ser detectadas por essa avaliação. Assim, as correlações menos significantes encontradas neste estudo, em relação a TG e HDL-colesterol, quando comparadas às demais variáveis antropométricas, provavelmente seriam elucidadas pelo fato de que essa avaliação demonstra uma estimativa relativamente baixa da massa gorda em indivíduos mais velhos ${ }^{35,36}$.

O estudo de Souza e cols..$^{31}$ revelou tendência, para ambos os sexos, de os indivíduos com elevadas medidas de CC apresentarem CT e TG elevados (acima de 240,0 e 200,0, respectivamente). Lakka e cols. ${ }^{37}$ examinaram a variância entre os tercis da CC e da RCQ com o perfil lipídico de homens adultos, demonstrando que quanto maior a obesidade global maiores são os valores de TG e menores os valores de HDL-colesterol. Esse estudo apresenta similaridades com os resultados encontrados nesta pesquisa, em que a obesidade corporal, avaliada por $\Sigma D C$, IMC, CC e RCQ, foi diretamente associada aos níveis de TG e inversamente associada aos níveis de HDL-colesterol. Contudo, a CC e a RCQ obtiveram nível de significância mais elevado ( $p<0,001)$.

Resultados mais precisos e similares aos aqui encontrados são observados na investigação realizada por Cabrera e Jacob Filho $^{38} \mathrm{em}$ mulheres idosas brasileiras. A grande prevalência de níveis baixos de HDL-colesterol foi encontrada nas mulheres com elevados valores de massa gorda central $(38,0 \%$ para a RCQ $>$ 1,01 e 35,6\% para a circunferência abdominal $[C A]>100 \mathrm{~cm}$, ambos com $p<0,05)$, sugerindo relação inversa entre obesidade central e HDL-colesterol. Além disso, a relação direta entre obesidade central e TG também coincide com os resultados aqui apresentados. A prevalência de obesidade central foi de 28,6\% quando analisada pela RCQ e de $27,9 \%$, pela CA.

A relação mais significante entre CC e RCQ com os componentes do perfil lipídico pode ser explicada pelo fato de a obesidade central estar diretamente ligada à gordura visceral, a qual, por sua vez, é um indicador de alterações metabólicas desfavoráveis. Tais efeitos influenciam o desenvolvimento aterogênico e a hipertensão, assim como acarretam alterações na resistência à insulina, na intolerância à glicose e na hipertrigliceridemia, elevam o índice de LDL-colesterol e reduzem o índice de HDL-colestero ${ }^{33,37,38}$. 
Além disso, o grande número de mulheres com baixos valores de HDL-colesterol (91,1\%), em que 62,5\% apresentaram valores de risco moderado entre 40,0 mg/dl e 60,0 mg/dl e $27,6 \%$ apresentaram risco elevado abaixo de $40,0 \mathrm{mg} / \mathrm{dl}$, sugere que esses indivíduos apresentam elevado risco aterogênico, em decorrência da associação inversa entre os níveis dessa lipoproteína e o risco para aterosclerose em indivíduos com mais de 50 anos de idade ${ }^{39,40}$. Esse efeito antiaterogênico ou ateroprotetivo apresenta-se bem definido, em que o aumento em cada $1 \mathrm{mg} / \mathrm{dl}$ do HDL-colesterol reduz em aproximadamente $2 \%$ a $3 \%$ o risco para doenças coronarianas ${ }^{39,41,42}$. De forma semelhante, o aumento de um desvio padrão no HDL-colesterol diminui a probabilidade de novos eventos cardiovasculares em $15 \%(p=0,015)$ em homens ${ }^{43}$.

Limitações - A amostra estudada foi constituída de mulheres idosas participantes de grupos comunitários na cidade de Curitiba, Paraná. Dessa forma, este estudo não atingiu indivíduos com graus de dependência mais acentuada, os quais em geral permanecem apenas no ambiente familiar. Os resultados obtidos demonstram uma tendência a que o excesso de obesidade central acarrete alterações negativas no perfil lipídico para essa população específica, aconselhando-se, portanto, que novos estudos sejam realizados, principalmente em mulheres após os 50 anos de idade, a fim de examinar essas associações, contribuindo para um diagnóstico precoce desses fatores de risco para doenças cardiovasculares.

\section{Conclusões}

De acordo com os resultados do presente estudo, as mulheres que apresentaram excesso de massa gorda na região central demonstraram alterações no lipidograma, principalmente reduções do HDL-colesterol. A análise de

\section{Referências}

1. Camarano AA. O envelhecimento da população brasileira: uma contribuição demográfica. Rio de Janeiro: Instituto de Pesquisa e Estatística Aplicada - IPEA; 2002. p. 1-97.

2. Stangl V, Baumann G, Stangl K. Coronary atherogenic risk factor in women. Eur Heart J. 2002; 23: 1738-52.

3. Smith Jr SC, Allen J, Blair SN, Bonow RO, Brass LM, Fonarow GC, et al. AHA/ ACC Guidelines for secondary prevention of patients with coronary and other atherosclerotic vascular diseases: 2006 update. Endorsed by the National Heart, Lung, and Blood Institute. Circulation. 2006;113: 2363-72.

4. Taddei CFG, Franken RA. Aterosclerose: fisiopatologia e prevenção de fatores de risco. In: Freitas EV, Py L, Neri AL, Cançado FAX, Gorzoni ML, Rocha M. Tratado de geriatria e gerontologia. Rio de Janeiro: Guanabara Koogan; 2002. p. 228-32.

5. Zhu S, Heshka S, Wang Z, Shen W, Allison DB, Ross R. Combination of BMI and waist circumference, and health risk for identifying cardiovascular risk factors in whites. Obes Res. 2004;12:633-45.

6. Boyle EM, Lille ST, Allaire E, Clowes AW, Verrier ED. Atherosclerosis. Ann Thorac Surg. 1997;64:47-56.

7. Wilson PW, Hoeg JM, D'Agostino RB, Silbershatz H, Belanger AM, Poehlmann $\mathrm{H}$, et al. Cumulative effects of high cholesterol levels, high blood pressure, and cigarette smoking on carotid stenosis. N Engl J Med. 1997;337:516-22.

8. Ross R. The pathogenesis of atherosclerosis. In: Braunwald E. Heart disease: a textbook of cardiovascular medicine. 4th ed. Philadelphia, PA: W. B. Saunders Company; 1992. correlação parcial e a maior variância encontrada na CC e na $\mathrm{RCQ}$, quando comparada aos demais fatores de risco indireto e aos fatores de risco direto, componentes do lipidograma, sugerem que ambos os métodos podem auxiliar o diagnóstico precoce em relação aos efeitos metabólicos desfavoráveis que o acúmulo de gordura corporal pode acarretar, como o desenvolvimento da aterosclerose.

\section{Agradecimentos}

Os autores agradecem a Dra. Mauren Isfer, bioquímica responsável pela realização das dosagens sangüíneas no laboratório de analises clínicas do Hospital de Polícia do Paraná. Ao Projeto DOCE - Diabetes Objetivando Controle e Educação - pela parceria realizada juntamente com o laboratório NOVARTIS, Farmácia DAS SETE. Às endocrinologistas do Serviço de Endocrinologia e Diabetes do Hospital Universitário Evangélico de Curitiba - Dras. Sheyla Q. Alonso, Carolina F da Luz Martins, Gabriela C. de Mira e Jeanne D. Gama, pela colaboração na coleta de dados.

\section{Potencial Conflito de Interesses}

Declaro não haver conflitos de interesses pertinentes.

\section{Fontes de Financiamento}

O presente estudo não teve fontes de financiamento externas.

\section{Vinculação Acadêmica}

Hão há vinculação desse estudo a programas de pósgraduação.

9. Cotran RS, Munro JM. Pathogenesis of atherosclerosis: recent concepts. In: Grundy SM, Bearn AG. The role of cholesterol in atherosclerosis. Philadelphia, PA: Hanley \& Belfus, Inc.; 1987.

10. Tankó LB, Bagger YZ, Qin G, Alexandersen P, Larsen PJ, Christiansen C. Enlarged waist combined with elevated triglycerides is a strong predictor of accelerated atherogenesis and related cardiovascular mortality in postmenopausal women. Circulation. 2005;111:1883-90.

11. De Michele M, Panico S, lannuzzi A, Celentano E, Ciardullo AV, Galasso R, et al. Association of obesity and central fat distribution with carotid artery wall thickening in middle-aged women. Stroke. 2002;33:2923-8.

12. Takami R, Takeda N, Hayashi M, Sasaki A, Kawachi S, Yoshino K, et al. Body fatness and fat distribution as predictors of metabolic abnormalities and early carotid atherosclerosis. Diabetes Care. 2001; 24 (7): 1248-52.

13. Despres JP, Morjani S, Lupien PJ, Tremblay A, Nadeau A, Bouchard C. Regional distribution of body fat, plasma lipoproteins, and cardiovascular disease. Atherosclerosis. 1990;10:497-511.

14. Cabrera MAS, Wajngarten M, Gebara OCE, Diament J. Relação do índice de massa corporal, da relação cintura-quadril e da circunferência abdominal com a mortalidade em mulheres idosas: seguimento de cinco anos. Cad Saúde Pública. 2005;21(3):767-75.

15. Cabrera MAS, Gebara OCE, Diament J, Nussbacher A, Rosano G, Wajngarten M. Metabolic syndrome, abdominal obesity, and cardiovascular risk in elderly women. Int J Cardiol. 2007;114(2):224-9.

16. Ferreira MG, Valente JG, Gonçalves-Silva RMV, Sichieri R. Acurácia da 
circunferência da cintura e da relação cintura/quadril como preditores de dislipidemias em estudo transversal de doadores de sangue de Cuiabá, Mato Grosso, Brasil. Cad Saúde Pública. 2005;22(2):307-11.

17. Wannamethee SG, Shaper AG, Walker M. Overweight and obesity and weight change in middle aged men: cardiovascular disease and diabetes. J Epidemiol Community Health. 2005;59:134-9.

18. Zamboni M, Mazzalli G, Zoico E, Harris TB, Meigs JB, Di Francesco V, et al. Health consequences of obesity in the elderly: a review of four unresolved questions. Int J Obes. 2005;29:1011-29.

19. Lemos-Santos MGF, Valente JG, Gonçalves-Silva RMV, Sichieri R. Waist circumference and waist-to-hip ratio as predictors of serum concentration of lipids in Brazilian men. Nutrition. 2004;20:857-62.

20. Lohman TG, Roche A, Martorell R (eds.). Anthropometric standardization reference manual. Abridged Edition. Champaign, IL: Human Kinetics; 1988.

21. Heyward VH, Stolarczyk LM. Anthropometric method applied body composition assessment. Champaign, IL: Human Kinetics; 1996.

22. Jackson AS, Pollock ML. Practical assessment of body composition. Physic Sportsmed. 1985;13:76-90.

23. Friedewald WT, Levy RI, Fredrickson DS. Estimation of the concentration of low-density lipoprotein in plasma without use of the preparative ultracentrifuge. Clin Chem. 1972;18:499-502.

24. Bassett MT. Diabetes is epidemic. Am J Public Health. 2005;95 (9):1496.

25. Mokdad AH, Ford ES, Bowman BA, Dietz WH, Vinicor F, Bales VS, et al. Prevalence of obesity, diabetes, and obesity-related health risk factors, 2001. JAMA. 2003;289:76-9.

26. Horani MH, Mooradian AD. Management of obesity in the elderly. Treat Endocrinol. 2002;1:387-98.

27. Abate N. Obesity and cardiovascular disease: pathogenetic role of the metabolic syndrome and therapeutic implications. J Diabetes Complications. $2000 ; 14: 154-74$

28. Obesity: preventing and managing the global epidemic. Report of a WHO consultation. World Health Organ Tech Rep Ser. 2000;894:1-253.

29. Lean MEJ, Han TS, Morrison CE. Waist circumference as a measure for indicating need for weight management. BMJ. 1995;311:158-61.

30. Romero-Corral A, Montori VM, Somers VK, Korinek J, Thomas RJ, Allison TG, et al. Association of bodyweight with total mortality and with cardiovascular events in coronary artery disease: a systematic review of cohort studies. Lancet. 2006;19:624-5

31. De Souza LJ, Souto Filho JTD, De Souza TF, Reis AFF, Gicovate Neto C,
Bastos DA, et al. Prevalence of dyslipidemia and risk factors in Campos dos Goytacazes, in the Brazilian State of Rio de Janeiro. Arq Bras Cardiol. 2003;81(3):257-64.

32. Pascot A, Lemieux I, Prud'homme D, Tremblay A, Nadeau A, Couillard C et al. Reduced HDL particle size as an additional feature of the atherogenic dyslipidemia of abdominal obesity. J Lipid Res. 2001;42:2007-14.

33. Sociedade Brasileira de Cardiologia. III Diretrizes Brasileiras sobre Dislipidemias e diretriz de prevenção da aterosclerose do Departamento de Aterosclerose. Arq Bras Cardiol. 2001;77 (supl 3): 1-48.

34. Hu D, Hannah J, Grey RS, Jablonski KA, Henderson JA, Robbins DC, et al. Effects of obesity and body fat distribution on lipids and lipoproteins in nondiabetic American Indians: The Strong Heart Study. Obes Res. 2000;8:411-21.

35. Brundavani V, Murthy SR, Kurpad AV. Estimation of deep-abdominal-adiposetissue (DAAT) accumulation from simple anthropometric measurements in Indian men and women. Eur J Clin Nutr. 2006;60:658-66.

36. Hughes VA, Roubenoff R, Wood M, Frontera WR, Evans WJ, Singh MAF. Anthropometric assessment of 10-y changes in body composition in the elderly. Am J Clin Nutr. 2004;80:475-82.

37. Lakka TA, Lakka HM, Salonen R, Kaplan GA, Salonen JT. Abdominal obesity is associated with accelerated progression of carotid atherosclerosis in men. Atherosclerosis. 2001;154:497-504

38. Cabrera MAS, Jacob Filho W. Obesidade em idosos: prevalência, distribuição e associação com hábitos e co-morbidades. Arq Bras Endocrinol Metab. 2005;45(5):494-501

39. Nambi V, Ballantyne CM. Fat, fit, and leading the charge. The evaluation of measuring high-density lipoprotein subpopulations. Arterioscler Thromb Vasc Biol. 2005;25:2013-5

40. Lessa I, Conceição JL, Souza ML, Oliveira V, Carneiro J, Melo J, et al. Prevalência de dislipidemias em adultos da demanda laboratorial de Salvador Brasil. Arq Bras Cardiol. 1997;69(6):395-400.

41. Berglund L. Lipoprotein metabolism: a well-tried tool to characterize dyslipidemic mechanisms. Arterioscler Thromb Vasc Biol. 2006;26:1201-3.

42. Gordon DJ, Probstfield JL, Garrison RJ, Neaton JD, Castelli WP, Knoke JD, et al. High-density lipoprotein cholesterol and cardiovascular disease: four prospective American studies. Circulation. 1989;79:8-15.

43. Rubins HB, Robins SJ, Collins D, Fye CL, Anderson JW, Elam MB, et al., for the Veterans Affairs High-Density Lipoprotein Cholesterol Intervention Trial study group. Gemfibrozil for the secondary prevention of coronary heart disease in men with low levels of high-density lipoprotein cholesterol. N Engl J Med. 1999;341:410-8. 